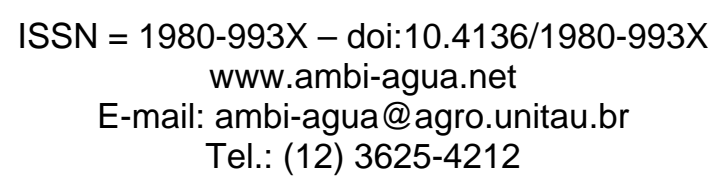

\title{
Aspectos conceituais sobre o regime hidrológico para a definição do hidrograma ambiental (doi:10.4136/ambi-agua.179)
}

\author{
Paulo da Costa Medeiros"; Francisco de Assis Salviano de Sousa ${ }^{2}$; \\ Márcia Maria Rios Ribeiro ${ }^{2}$ \\ ${ }^{1}$ Universidade Federal de Campina Grande - UFCG \\ E-mail: medeiros_pc@yahoo.com.br \\ ${ }^{2}$ Centro de Tecnologia e Recursos Naturais \\ E-mail: fassis@dca.ufcg.edu.br; mm-ribeiro@uol.com.br
}

\section{RESUMO}

Considerando os instrumentos da Política Nacional de Recursos Hídricos Brasileira, a Lei № 9.433 de 1997 confere à outorga dos direitos de uso da água, a limitação ao usurário de água de determinada vazão nos reservatórios superficiais e/ou subterrâneos. O somatório das vazões outorgadas não deverá afetar as quantidades limites que proporcionem as condições ecológicas naturais dos rios (vazão ecológica). Conciliar a gestão dos recursos hídricos e ambientais representa um complexo desafio pois envolve muitas áreas de conhecimento como a ecologia e a hidrologia. O presente artigo enfatiza a necessidade de uma maior aproximação entre essas áreas por meio da interpretação espaço-temporal das limitações hídricas nos regimes naturais de vazões. Esses regimes podem ser observados nos hidrogramas, indispensáveis ferramentas para uma melhor compreensão do comportamento hidroecológico dos rios.

Palavras-chave: outorga dos direitos de uso da água; vazão ecológica; hidrograma.

\section{Conceptual aspects of the hydrological regime for the definition of environmental hydrograph}

\begin{abstract}
Considering the instruments established by the Brazilian National Policy for Water Resources (Law 9,433/1997), the water right concession has the aim to limit water use of surface and groundwater reservoirs. In this sense, the sum of all granted uptake rights must take into consideration the natural ecological flows. It has been a complex challenge to establish a trade off between the management of water and environmental resources since various aspects have to be considered involving many knowledge areas such as ecology and hydrology. This paper focuses on the need for a closer dialogue between these areas by considering the space-time interpretation of the restrictions on natural flow regimes of the water resources. These regimes can be observed in hydrographs, which are indispensable tools for better understanding the hydro-ecologic behavior of rivers.
\end{abstract}

Keywords: water right concession; ecological flow; hydrograph.

\section{INTRODUÇÃO}

Entre os frequentes conflitos que atualmente ocorrem no âmbito do uso dos recursos ambientais, seja por alterações naturais e/ou atividades antrópicas, está a necessidade do controle da água em sua quantidade e qualidade. Essa necessidade implica a responsabilidade da preservação dos corpos hídricos, em harmonia com a fauna e a flora, proporcionando qualidade de vida para as gerações atuais e futuras. 
MEDEIROS, P. C.; SOUZA, F. A. S.; RIBEIRO, M. M. R. Aspectos conceituais sobre o regime hidrológico para a definição do hidrograma ambiental. Ambi-Agua, Taubaté, v. 6, n. 1, p. 131-147, 2011. (doi:10.4136/ambi-agua.179)

Dentre os instrumentos de gestão da Política Nacional de recursos hídricos, a Lei ${ }^{\circ}$ 9.433 de 1997 (Brasil, 1997) confere a outorga dos direitos de uso da água, a limitação ao usurário de água de determinada vazão nos reservatórios superficiais e/ou subterrâneos. O somatório das vazões outorgadas nos diversos tipos de uso/usuários não deverá afetar as quantidades limites que proporcionem as condições ecológicas naturais dos rios. Várias são as dificuldades encontradas na implementação desse instrumento de gestão, dentre elas os fatores técnicos (sistema de outorga), notadamente na definição dos critérios para a outorga.

Conciliar limitações hídricas ambientais e as demandas nos diversos tipos de usos e usuários tem sido tarefa complexa e desafiadora, envolvendo vários aspectos (socioeconômicos, físicos, químicos, biológicos, culturais, ambientais, entre outros). Dentre esses, considerando o biológico e o comportamento espaço-temporal dos corpos hídricos, verifica-se a necessidade de discussões e maior aproximação entre as áreas ecológica e hidrológica, entre teorias, conceitos e banco de dados, harmonizando maior afinidade entre a realidade hídrica e ambiental nas bacias hidrográficas.

De uma maneira geral, as dificuldades na relação entre a hidrologia e ecologia ainda são grandes (Carlisle et al., 2009), a recente ênfase de estudos relacionando os recursos hídricos e o meio ambiente tem mudado as prioridades de estimativas seguras do regime natural de vazões. Nos últimos anos, órgãos financiadores estão fomentando pesquisas com objetivo de subsidiar estudos sobre o referido tema compatibilizando os aspectos hidrológicos, limnológicos, ecológicos e socioeconômicos dos regimes de vazões (CNPq, 2006).

O presente trabalho fixa a discussão no aspecto hidrológico (vazões), por meio da interpretação das limitações hídricas no regime natural do escoamento dos rios, testemunhadas nos hidrogramas (que contemplam todos os aspectos físicos de uma bacia hidrográfica numa seção fluvial) como ferramenta fundamental para uma melhor compreensão do comportamento dos rios no contexto da hidrobiologia/eco-hidrologia.

\section{VAZÃO FLUVIAL: CONTEXTO ECOLÓGICO E DE GESTÃO}

\subsection{O fluxo de água e a ecologia dos rios}

Para Eamus et al. (2006) eco-hidrologia é o estudo integrado da água e vegetação ripária, que requer informações ecofisiológicas, hidrológicas, geológicas e micrometeorológicas. Ao longo de todo o percurso do rio, diferentes sedimentos vegetais das zonas ribeirinhas fornecem nutrientes orgânicos fundamentais para os ecossistemas que sistematicamente alimentam o perímetro do canal fluvial da cabeceira à foz (Weller et al., 1998; Minshall e Rugenski, 2007). Os rios são ecossistemas abertos, interagindo atmosfera-água-terra, os componentes de entrada (vazão de entrada, escoamento lateral, precipitação, infiltração e os rios efluentes) e de saída (vazão escoada; evaporação, evapotranspiração e o fluxo nos rios influentes) resumem as principais situações de movimento da água associado ao escoamento nos períodos chuvosos e de estiagem. Destaca-se a importância das dimensões espacial (vertical, longitudinal e lateral) e temporal, devendo serem observadas mudanças físicas, químicas e biológicas, processos hidrológicos e geomorfológicos, ocasionados por fenômenos naturais ou por intervenção humana (Petts 2000; Brown e Pasternack, 2008; Hu et al., 2008).

Na dimensão longitudinal do rio, observa-se o conceito de “Contínuo Fluvial” proposto por Vannote et al. (1980), bastante citado na literatura eco-hidrológica para ecossistemas em movimento (Meyer, et al., 2003; Ciesielka e Bailey, 2007; Gore, 2007; Jørgensen et al., 2007; Callanan et al., 2008; Boulton et al., 2008; Burcher, 2009), que considera dentre outros aspectos, a contínua mudança nas condições ambientais do fluxo de primeira ordem para ordens superiores, dividindo o rio em três regiões geomorfológicas distintas: região de nascente (alta contribuição terrestre de sedimentos orgânicos), médio curso (mais relacionado com a produção de algas e plantas aquáticas) e proximidade da foz (disposiçãoà turbidez, carga elevada de sedimentos procedentes de montante). Montgomery (1999), no conceito de 
MEDEIROS, P. C.; SOUZA, F. A. S.; RIBEIRO, M. M. R. Aspectos conceituais sobre o regime hidrológico para a definição do hidrograma ambiental. Ambi-Agua, Taubaté, v. 6, n. 1, p. 131-147, 2011. (doi:10.4136/ambi-agua.179)

“Domínio de Processos”, destaca aspectos físicos como clima, geologia, topografia, entre outros, determinantes nas características dos habitats, influenciando os processos dos ecossistemas ribeirinhos.

Nos estuários, a exemplo de Miller e Ullman (2004), Hays e Ulman (2007), atenta-se ao papel ecológico substancial, em que as taxas de salinidade intersticiais variam com a sazonalidade (Bruno et al., 2001), favorecendo ciclo de vida adaptável ao equilíbrio das águas na região costeira. Tais ambientes apresentam aspectos biológicos e físico-químicos bastante complexos (Hardisty, 2007) e vulnerabilidade aos efeitos das marés (Zedler, 2001).

Nas dimensões lateral e vertical do rio, aspectos geomorfológicos dos solos e ações antrópicas (Renschler et al., 2007), como poluição e desmatamento na mata ciliar, são fortes influenciadores na qualidade e quantidade da água. A zona hiporreica, referente a interface em que ocorre a "ligação" (mistura) da água superficial com as águas subterrâneas situada abaixo ou ao lado do canal fluvial (Alley et al., 2002; McClain et al., 2003), é de grande importância para o ciclo de energia, nutrientes (Postel e Richter, 2003). Um bom exemplo da fauna abundante nessas zonas são os copépodes (Galassi, 2001; Reid, 2001), seres crustáceos que vivem nos diversos tipos de aquíferos, que auxiliam a cadeia alimentar dos peixes ao longo do rio. Ressalta-se que o desenvolvimento e aplicação de metodologias sobre ressurgências (fluxo aquífero-rio) são bastante laborais, de difícil mensuração (Kalbus et al., 2006; Brodie et al., 2007a; Cey et al., 2008; Luz et al., 2008; Rosenberry, 2008; Buss et al., 2009).

A significativa relação sazonal do rio com a ecologia pode ser observada em Postel e Richter (2003) que apresentam funções ecológicas (na fauna e flora ripárias) em diferentes níveis de vazões, discretizando em: vazões baixas (fluxo basal) - nível de seca; vazões baixas - nível normal; vazões altas e grandes inundações.

\subsection{A vazão ecológica}

Cruz (2000 apud Cruz, 2005) define a vazão ecológica como "necessária para que sejam preservadas as condições de pulso hidrológico, transporte de sedimentos e nutrientes, sincronicidade com o ciclo de vida das espécies silvestres da fauna e da flora e a taxa de perturbações necessárias à renovação e funcionamento dos ecossistemas associados ao curso de água, ou seja, a vazão necessária para manter as funções que mantêm o mosaico de biótopos que compõem o rio, nos seus leitos maiores e menores”. Para o caso do semiárido, Luz et al. (2007) destacam que "o conceito de vazão ecológica não pode ser plenamente aplicado a rios intermitentes devido às diferenças entre a natureza destes e as metodologias empregadas, bem como os aspectos operacionais”.

Considerando o mosaico de manchas de habitats na heterogeneidade espacial ao longo do rio, bem como a heterogeneidade temporal como resposta do sistema para o regime de perturbações (Benda et al., 2004), pode-se definir a vazão ecológica como sendo o volume de água, ao longo das mudanças espaço-temporais, necessário à preservação dos ecossistemas relacionados com o corpo hídrico.

Alves e Bernardo (2000) e Tharme (1996 apud Cruz, 2001) apresentam três métodos para a quantificação das vazões ecológicas: hidrológicos (vazões mínimas baseadas nas curvas de permanência ou curvas de frequências de vazões para diversas durações), que são amplamente utilizados no Brasil, como Queiroz et al. (2005) e Santos et al. (2006), como também bastante contestados como em Silva et al. (2006), Farias Júnior et al. (2005); hidráulicos (relações entre várias variáveis hidráulicas tais como, perímetro molhado, máxima profundidade em função da vazão e fatores ambientais limitantes nos zonas riparias); e padrões de habitat (considera os aspectos dos micro-habitats ao longo do curso d’água com as mudanças de descargas).

Collischonn et al. (2005) destacam a insuficiência do tradicional critério de vazão ecológica como um valor único, bem como os critérios de definição de vazão remanescente 
MEDEIROS, P. C.; SOUZA, F. A. S.; RIBEIRO, M. M. R. Aspectos conceituais sobre o regime hidrológico para a definição do hidrograma ambiental. Ambi-Agua, Taubaté, v. 6, n. 1, p. 131-147, 2011. (doi:10.4136/ambi-agua.179)

nos rios que devem contemplar não apenas as situações de vazões mínimas durante os períodos de estiagem, mas também os outros períodos que caracterizam o regime hidrológico, como a discretização sazonal (Moliere et al., 2009). Um método que utiliza informações hidráulicas e os padrões do habitat é o "Instream Flow Incremental Methodology - IFIM”. O IFIM estabelece cinco fases no seu emprego (identificação do problema; planejamento de estudo; implementação do estudo; análise de alternativas e resolução de problemas) exigindo uma gama de informações e tempo para coleta de dados (Benetti et al., 2003).

Cruz (2005) comenta que os modelos para determinação da vazão ecológica, atualmente utilizados, estão direcionados à visão praticada desde a década de 1950 (estabelecimento de vazões remanescentes mínimas). Os biólogos da década de 1970 e 1980 acreditavam que vazões elevadas em períodos de secas garantiriam melhor qualidade de vida aos peixes. Entretanto, na visão atual, observa-se que os modelos que se aproximam do regime de fluxo natural de um rio, garantem melhor qualidade de vida para flora e fauna aquáticas (Chang et al., 2008, Sanderson et al., 2008; Deitch et al., 2009a), pois vazões altas podem beneficiar determinada espécie, mas comprometer outras e vice-versa (King et al., 2008).

Para outros autores a vazão ecológica infere também no aspecto social e econômico, a exemplo do semiárido nordestino brasileiro, onde a necessidade hídrica está estritamente relacionada às comunidades ribeirinhas que utilizam a água como estratégia de sobrevivência, escavando "cacimbas" (poços amazonas rasos) no leito dos rios e levando animais às vegetações ripárias nos períodos de seca (Maltchik, 1999).

O Banco Mundial (2003 apud Farias Júnior, 2006), considera um conceito mais amplo referindo-se aos usos humanos integrados às demandas ambientais, recebendo a denominação de vazão ambiental, "onde se avalia e integra os interesses de todos os componentes do ecossistema”. Medeiros et al. (2006) utilizam o conceito de vazão ambiental, contemplando os usos múltiplos da água bem como o atendimento das demandas dos ecossistemas aquáticos e terrestres dependentes da sazonalidade hidrológica.

Observa-se que a grande maioria dos trabalhos pouco atenta para relação das águas subterrâneas no sistema hidrológico dos rios, mesmo com o crescimento da exploração dessas águas no mundo, uma atenção secundária tem sido dada em termos políticos e legais comparados com as águas superficiais (Eckstein e Eckstein, 2005). O "losing stream” e o “gaining stream” citado por Tood (1987), Feitosa (1997), Winter et al. (1998), Leap (1999), Fitts (2002), Pinder e Celia (2006), Brodie et al. (2007b) mostram as relações que abrangem os períodos de estiagens aos períodos de cheias, mediante armazenamento e descarga nas margens ripárias. A carência de banco de dados (Smakhtin, 2001), as lacunas na compreensão dos processos que envolvem a referida interconexão, bem como a retirada de água sem gestão para as diversas demandas, pode comprometer de maneira irreversível o equilíbrio ambiental tendo em vista o importante papel hidrogeológico no sistema (Harvey et al., 2007).

\section{3. Águas outorgáveis e não outorgáveis frente ao potencial hídrico na bacia hidrográfica}

A crescente necessidade do uso racional da água vem sendo fundamentalmente condicionada pelos fatores socioeconômicos e alterações hidroclimatológicas no planeta (por causas naturais e/ou antropogênicas). As estimativas das quantidades hídricas para o atendimento das demandas de água estão limitadas principalmente às prioridades de provimento de água: o abastecimento humano e a preservação dos ecossistemas (vazão ambiental).

Albuquerque e Rêgo (1998) discretizam o ciclo hidrológico em vários sub-sistemas que interagem com a água, tais como o proposto na Figura 1. Na Figura observa-se o escoamento dos rios (saldo da precipitação menos as perdas pela evapotranspiração real) no sistema hidrográfico, subdividindo-se em superficial e de base, ambos participantes nas fases de recarga e descarga das águas nos aquíferos conexos, em seus níveis: acima, o freático (ou 
MEDEIROS, P. C.; SOUZA, F. A. S.; RIBEIRO, M. M. R. Aspectos conceituais sobre o regime hidrológico para a definição do hidrograma ambiental. Ambi-Agua, Taubaté, v. 6, n. 1, p. 131-147, 2011. (doi:10.4136/ambi-agua.179)

livre); abaixo, o subsistema confinado (limitado por rochas semi-impermeáveis havendo a troca de fluxo conforme pressões hidráulicas entre elas) - se o subsistema for litorâneo (ou costeiro), o escoamento poderá ocorrer diretamente no mar ou através de fluxo subsuperficial. Os autores apresentam algumas definições para avaliação e gerenciamento de conjunto de recursos hídricos superficiais e subterrâneos:

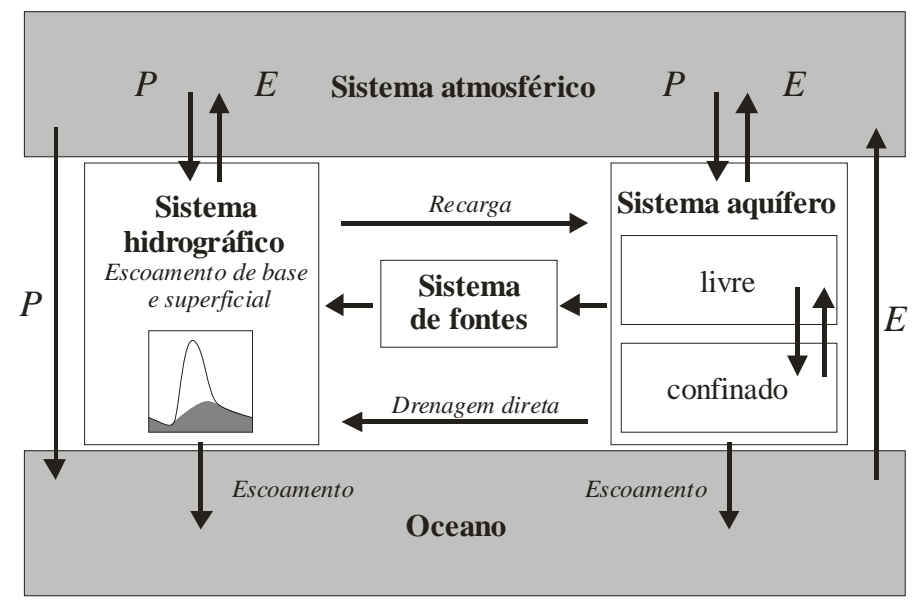

Figura 1. Alguns sistemas do ciclo hidrológico (adaptado de Albuquerque e Rêgo (1998)).

- O potencial: é definido como sendo a vazão de um rio ou aquífero, anualmente renovável. A mensuração do potencial dos recursos hídricos pode ser feita pela análise e quantificação de hidrogramas (áreas dos aportes superficial e subterrâneo, e a íntegra se traduz no escoamento fluvial). Se o sistema aquífero sofre influência do mar, considera-se ainda a contribuição sub-superficial do oceano considerando a Lei de Darcy, Conservação da Massa ou indiretamente, por meio de um Balanço Hídrico.

- As reservas: representam águas seculares - acumuladas em vazios entre os grãos dos minerais, não necessariamente renováveis ao ano, estratigraficamente (?) situam-se abaixo do potencial de modo que, se exploradas, evidenciam que este já tinha se esgotado, carreando problemas hidro-ecológicos, geotécnicos, entre outros, de amplitudes até calamitosas, tais como: compactação de aquíferos, comprometimento de vegetação ripária, intrusão salina/avanço da água do mar.

- As disponibilidades: representam o volume de água efetivamente disponível em termos de qualidade e quantidade, é o potencial subtraído das perdas e quantidades hídricas ecológicas, é dita máxima (máximo do potencial que pode ser convertido em disponibilidades) e atual (correspondente à capacidade de regularização da bacia frente as demandas existentes).

Diante do potencial hídrico de uma bacia hidrográfica, retirando-se as perdas do sistema e as quantidades hídricas associadas à preservação ambiental, a disponibilidade máxima de água seria destinada às demandas outorgadas e isentas de outorga, priorizando-se o abastecimento humano aos demais tipos de usos.

Atenta-se que a Lei 9.433/97 (Política Nacional de Recursos Hídricos), em seu Art. $12^{\circ}$ ( $\S 1^{\circ}$ ), destaca que não estão sujeitos à outorga casos como "derivações, captações, acumulações de volumes e lançamentos considerados insignificantes”. No entanto, o somatório dessas vazões "insignificantes" pode representar parte expressiva dos recursos hídricos do sistema, condicionando redução mais acelerada da disponibilidade hídrica atual que "mascara” o real somatório demandado do sistema hídrico na bacia hidrográfica.

A Figura 2 mostra as principais subdivisões hídricas no processo de outorga quantitativa, relacionando a facilidade e preferência de acesso às águas em suas ocorrências (superficial e 
MEDEIROS, P. C.; SOUZA, F. A. S.; RIBEIRO, M. M. R. Aspectos conceituais sobre o regime hidrológico para a definição do hidrograma ambiental. Ambi-Agua, Taubaté, v. 6, n. 1, p. 131-147, 2011. (doi:10.4136/ambi-agua.179)

subterrânea): (a) as prioridades de uso (vazão ambiental e o abastecimento humano) e disponibilidades (com demandas que comumente se apresentam crescentes); e (b) os aportes superficial e subterrâneo, onde se primazia o uso das águas superficiais (renováveis em maior frequência) frente às subterrâneas (de cunho estratégico).

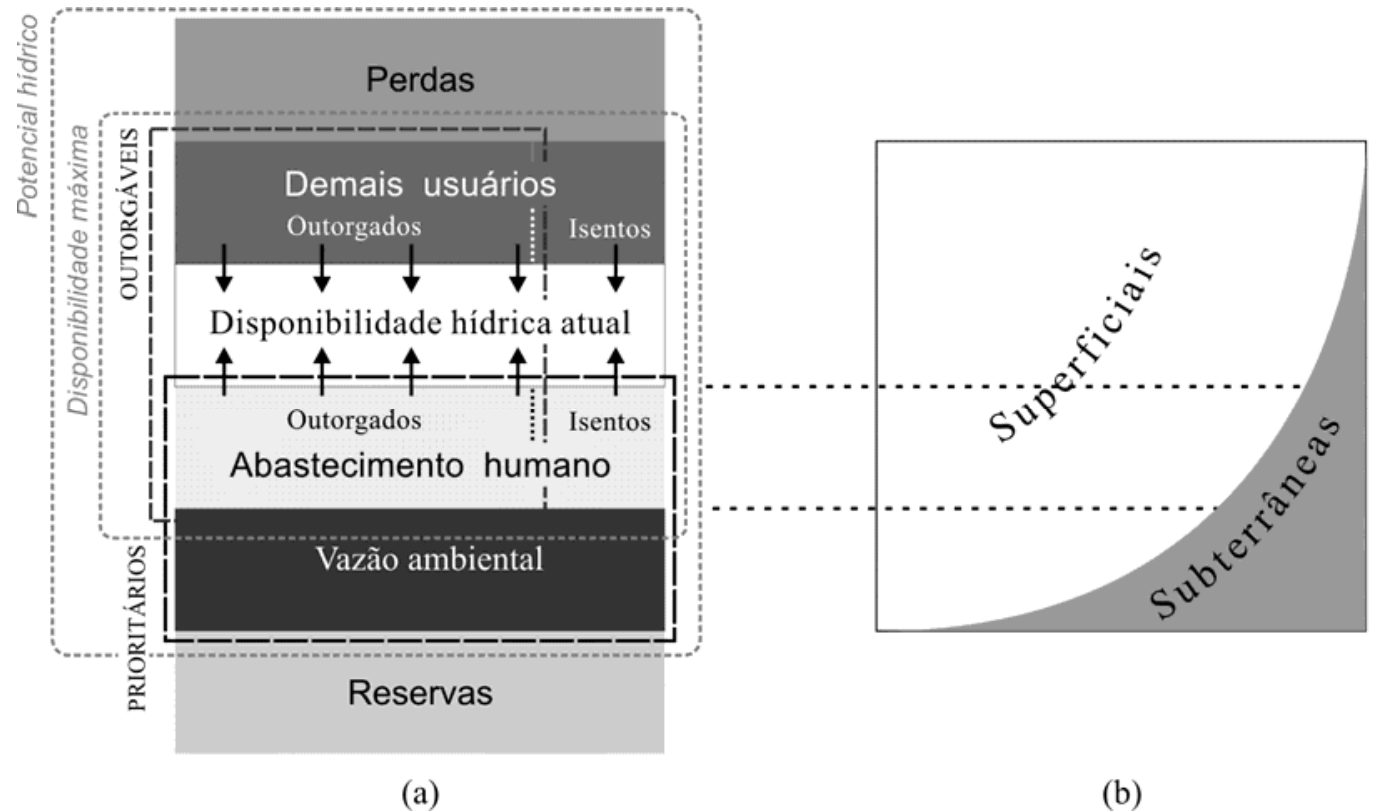

Figura 2. Principais subdivisões consideradas no processo de outorga do direito de uso da água: (a) aportes prioritários (a vazão ambiental e a quantidade de água sujeita à outorga); potencialidade e disponibilidade hídrica, enfatizando o crescente aumento das demandas (outorgadas e isentas de outorga) frente a quantidade hídrica disponível; (b) condição ideal da retirada da água bruta onde se primazia o uso das águas superficiais às subterrâneas. (autoria própria)

A falta de gestão dos recursos hídricos inibe melhorias sociais e econômicas, afetando direta e indiretamente o meio ambiente, em muitos casos, torna-se bastante complexo propor medidas que garantam o atendimento das demandas nos diversos tipos de usuários, como no Nordeste brasileiro, na região semiárida, onde frequentemente ocorrem secas severas e ainda são utilizadas "soluções" emergenciais e remediadoras (carros pipas e programas de beneficio financeiros a famílias de baixa renda) e no litoral, a exemplo da explotação de águas subterrâneas em zonas urbanas costeiras (funcionando em paralelo ao sistema de abastecimento público alimentado por mananciais superficiais), cujo excesso pode ultrapassar a recarga natural anualmente renovável, favorecendo a intrusão salina e desequilibrando o meio ambiente na região dos estuários).

\section{A ESCALA ESPAÇO-TEMPORAL E A VARIABILIDADE HIDROLÓGICA}

Para diferentes referenciais de observação, considerando-se os processos do ciclo hidrológico, uma área de estudo pode ser vista sob vários níveis, desde escalas globais (como a bacia hidrográfica e sistemas aquíferos), passando por modelos regionais (áreas que subdividem a bacia segundo critérios específicos ou em sub-bacias), considerações locais (poço ou uma microbacia industrial/rural, tomada a fio d'água, etc), ou mesmo ainda, a análise em duas ou mais amplitudes, como na da troca de fluxo de águas superficiais e subterrâneas (Dahl et al., 2007), que podem ser consideradas em escalas globais, regionais ou locais, relacionadas entre si por meio de diferentes fenômenos, interagindo de forma sistêmica em um único grande ciclo (Silva, 2007). Na mesma ótica, para a escala temporal, considerando-se seus níveis de observação, a análise mensal (descrevendo as sazões), por exemplo, poderia ser contextualizada com dados diários (para os pulsos de vazões) cujo 
MEDEIROS, P. C.; SOUZA, F. A. S.; RIBEIRO, M. M. R. Aspectos conceituais sobre o regime hidrológico para a definição do hidrograma ambiental. Ambi-Agua, Taubaté, v. 6, n. 1, p. 131-147, 2011. (doi:10.4136/ambi-agua.179)

"refinamento" estaria ligado a um interesse particular, como no comportamento dos habitats em determinado período do ano. Essas considerações bem como o tamanho e qualidade do banco de dados facilita bastante a confecção de modelos e seus níveis de gerenciamento, proporcionando melhores resultados que possam apoiar as tomadas de decisões dos gestores de recursos hídricos.

\subsection{O hidrograma discretizado}

O hidrograma contempla todo o desempenho hídrico da bacia, suas subdivisões retratam o comportamento da água no rio volumetricamente com o tempo e representa informação de grande utilidade para o planejamento dos recursos hídricos.

A separação dos componentes do hidrograma sempre tem sido um desafio na hidrologia (Nathan e McMahon, 1990, Kattan et al., 1987 apud Ferraz e Mortatti, 2003; Chen et al., 2006, Han e Hammond, 2006). Tucci (2003) cita a divisão do hidrograma composta por três componentes principais: a curva de ascensão, região de pico e o ramo de recessão. Este último reflete a contribuição das fontes subterrâneas de água nos períodos de estiagem, de comportamento bem mais complexo (Tallaksen, 1995) e bem mais dependente das variáveis do sistema, sendo bastante destacadas as subdivisões em recessão superior e inferior (Askoy, 2000). Jain e Srinivasulu (2006) apresentam divisões para o hidrograma mais discretizadas com ascendência em duas partes (segmento inicial e outro próximo ao cume sob maior influência do fluxo superficial, destacando-se que os processos físicos ocorrentes no primeiro são bastante diferenciados do segundo) e o ramo cadente em três partes (parte inicial nas proximidades do pico, parte mediana, influenciado pelo escoamento sub-superficial, e o segmento final - escoamento de base). A Figura 3 resume a separação do hidrograma adaptado de Shaw (2005) integrada às subdivisões citadas por Jain e Srinivasulu (2006), em que se destacam: o limite entre o escoamento de superfície e o de base (curva $a$ ) ao longo das sazões - de comportamento complexo bastante dependente da estrutura geológica e cobertura superficial da bacia; e o ponto (b) de início na curva do fluxo de base (tipicamente exponencial, melhor determinado no traçado da curva lnQ versus t).

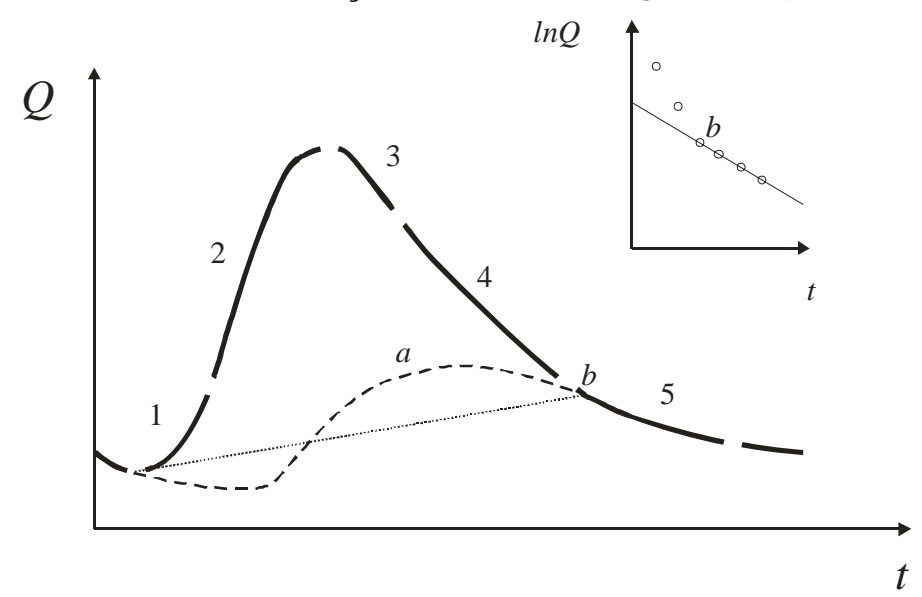

Figura 3. Subdivisão do hidrograma (Jain e Srinivasulu, 2006) e separação do escoamento de base, adaptado de Shaw (2005).

\subsection{O hidrograma no auxílio à definição da vazão ambiental}

Poff et al. (1997) citam que a variabilidade hidrológica é influenciada pela magnitude, duração, frequência e época de ocorrência de vazões mínimas e máximas (Jacobson e Galat, 2006; Neubauer et al., 2008; Masih et al., 2009; Gao et al., 2009; Wen, 2009; Zeilhofera e Moura, 2009), ilustrativamente observadas em Bunn e Arthington (2002) (Tucci 2009, Naiman et al., 2008), em que se destacam quatro princípios sobre a influência do regime natural de vazões na biodiversidade ripária mediante vários mecanismos relacionados no tempo e no espaço, resumidamente expressos em um hidrograma, atentando-se: às relações 
MEDEIROS, P. C.; SOUZA, F. A. S.; RIBEIRO, M. M. R. Aspectos conceituais sobre o regime hidrológico para a definição do hidrograma ambiental. Ambi-Agua, Taubaté, v. 6, n. 1, p. 131-147, 2011. (doi:10.4136/ambi-agua.179)

entre a diversidade ecológica e fluxo do canal (mudanças geomorfológicas pelas grandes vazões); a variabilidade hidrológica (sazonalidade); as trocas de fluxo vertical e lateral no escoamento (ressalta-se, mais uma vez, as supracitadas fases de rio efluente e influente) e as alterações no regime natural do fluxo quanto as espécies invasivas.

Destaca-se a necessidade de se conservar os níveis mínimos de fluxo de base (Weber e Perry, 2006; Deitch et al., 2009a; Deitch et al., 2009b), especialmente nas secas prolongadas, considerando-se as prioridades ecológicas, o comportamento do fluxo e a limitação/interrupção para explotação de água. Heicher (1993 apud Smakhtin, 2001), esboçou vários efeitos ambientais causados pela redução no escoamento de base (aumento da sedimentação, alterando a morfologia do canal, distribuição da biota, aumento da temperatura e penetração dos raios de sol, mudanças na densidade, produtividade, e composição de espécies).

A Figura 4 mostra alguns aspectos considerados para a confecção do hidrograma ambiental e o comportamento hídrico no contexto ecológico. Apresenta uma bacia fictícia, com pontos de controle sequencialmente distribuídos e respectivas seções fluviométricas com seus comportamentos hídricos no tempo e espaço, apresenta também as subdivisões do hidrograma, destacando o seccionamento das etapas do escoamento, sugerindo a outorga dos recursos hídricos (discretizada em águas superficiais e subterrâneas), enfatiza também a relevância de conflitos de uso em períodos de escassez hídrica. O limite mínimo do escoamento ambiental equivale "à média das vazões mínimas de cursos d’água perenes, capaz de assegurar a continuidade do regime da rede hidrográfica e, por conseguinte, da vida vegetal e animal dela dependente” (Costa et al., 2007).

\section{CONSIDERAÇÕES FINAIS}

A forte relação da variabilidade hidrológica e o meio ambiente aquático enfatiza a necessidade do hidrograma ambiental como instrumento que ajuda a esclarecer o comportamento da água em uma bacia hidrográfica ou trechos de rios, no contexto ecohidrológico (conservação ambiental dos corpos hídricos) e de gestão (atendimento aos múltiplos usos).

Alerta-se às dificuldades de acesso a banco de dados (quantidade, qualidade e disponibilidade), o que impede a integração com outras áreas de estudo, bem como a inércia no fornecimento de informações.

Destaca-se à necessidade de estudos de regime de fluxo dos rios (limites mínimos e máximos, frequências, amplitudes), de maneira a subsidiar os estudos ecológicos objetivando atender os níveis sazonais, a exemplo das subdivisões propostas por Postel e Richter (2003), os princípios alvitrados por Bunn e Arthington (2002).

Cruz (2005) destaca: "o regime de flutuação de fatores ambientais no tempo é reconhecido como um dos principais fatores do ambiente", e "no caso de ecossistemas aquáticos, o regime de flutuações das vazões e, consequentemente, das cotas de inundação, representa a principal função de força do sistema, regulando a heterogeneidade temporal e espacial da paisagem do rio”. Em sua metodologia apresentou análise espectral, gerando uma série sintética de pulsos de vazões usando-se a Transformada Rápida de Fourier em função de dados diários de fluxo, destacando-se esta técnica como bastante difundida para descrição das periodicidades em séries temporais.

Ideião et al. (2006) citam que além da Transformada de Fourier (TF) (mais popular), existem diversas transformações matemáticas que podem ser aplicadas, como a Transformada de Wavelet (TW) (ou ondaleta), sendo um avanço recente no processamento de sinais que tem atraído muita atenção em vários setores da ciência, "apropriada para analisar eventos irregularmente distribuídos e séries temporais que contêm potência não estacionária em diferentes frequências”. 
MEDEIROS, P. C.; SOUZA, F. A. S.; RIBEIRO, M. M. R. Aspectos conceituais sobre o regime hidrológico para a definição do hidrograma ambiental. Ambi-Agua, Taubaté, v. 6, n. 1, p. 131-147, 2011. (doi:10.4136/ambi-agua.179)

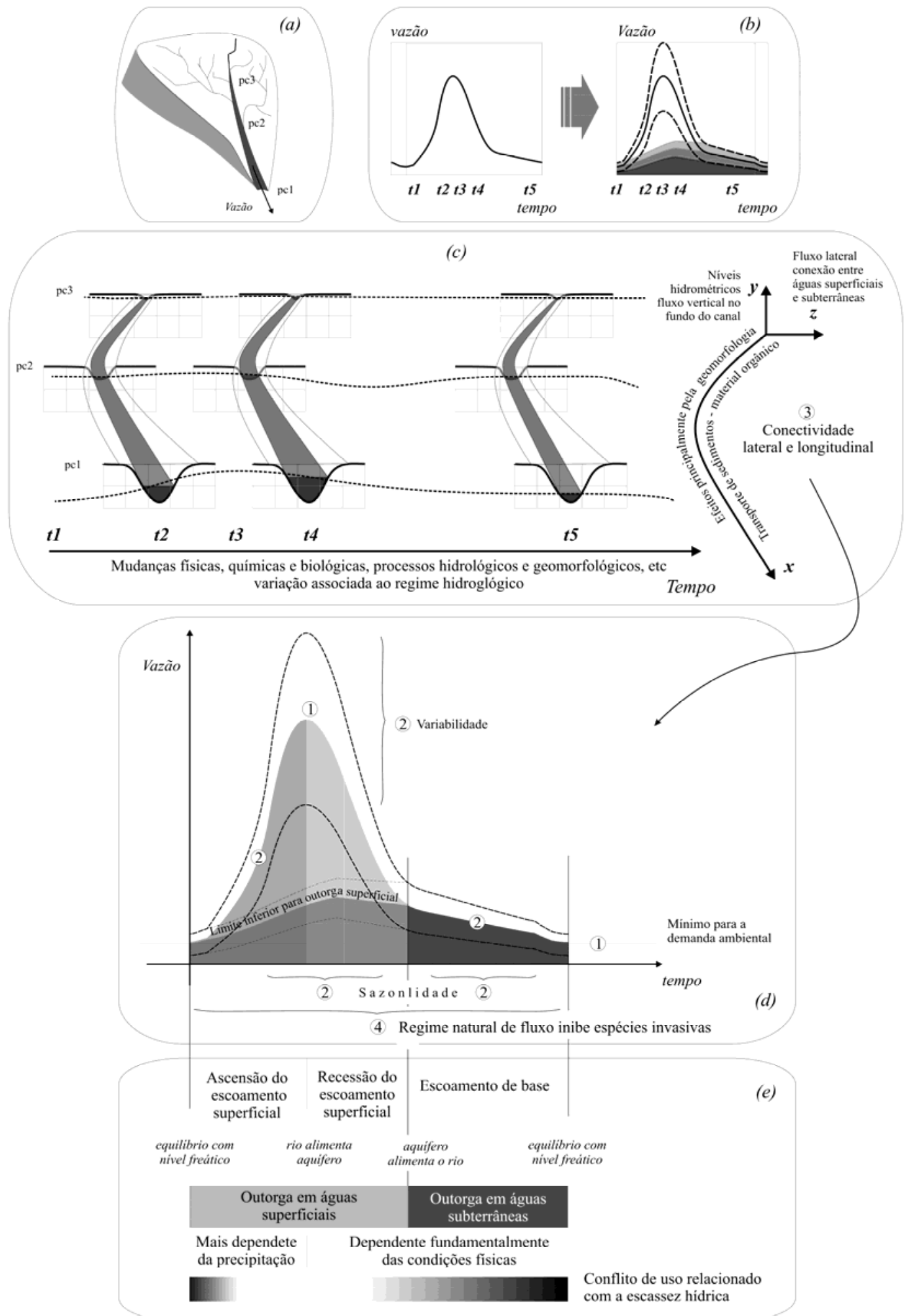

Figura 4. Alguns aspectos para a confecção do hidrograma ambiental e o comportamento hídrico no contexto ecológico: (a) pontos de controle; (b) variabilidade superficial/subterrânea; (c) variabilidade espacial (vertical, horizontal e longitudinal); e (d) Princípios do comportamento ecológico frente a variabilidade hidrológica (1-relações entre a diversidade ecológica e fluxo do canal; 2-sazonalidade; 3-trocas de fluxo vertical e lateral no escoamento; e 4-alterações no regime natural do fluxo quanto as espécies invasivas (adaptado de Bunn e Arthington (2002)); (e) aspectos da variabilidade na conexão rioaquífero (rios efluentes e influentes) (adaptado de Feitosa (1997)) e de gestão - limites de outorga (superficial e subterrânea) e risco de conflitos ante a escassez hídrica. (autoria própria). 
MEDEIROS, P. C.; SOUZA, F. A. S.; RIBEIRO, M. M. R. Aspectos conceituais sobre o regime hidrológico para a definição do hidrograma ambiental. Ambi-Agua, Taubaté, v. 6, n. 1, p. 131-147, 2011. (doi:10.4136/ambi-agua.179)

\section{CONCLUSÕES}

Diante da escassez de banco de dados eco-hidrológicos, a análise estatística de séries históricas de vazões, mesmo que, num contexto ambiental, a colocação seja limitada, pode contribuir significativamente para melhorias e desenvolvimento de futuros modelos, como no estudo das perturbações de fluxo, sensivelmente observadas na escala diária (mais intensas em períodos de cheias) e que são bastante relacionadas com o comportamento ecológico dos rios.

A concepção do hidrograma ambiental requer tempo e riqueza de dados, abrange várias disciplinas de pensamento interdisciplinar, com adaptações ao longo do tempo (como em comparações das situações anteriores e posteriores - das condições naturais às mudanças antropogênicas) e espaço (comparando as demandas hídricas à montante e à jusante de um eventual ponto de controle). A integração de profissionais e de metodologias numa amplitude multidisciplinar possibilitará maior aproximação da realidade ambiental dos rios, como na própria ideologia do desenvolvimento sustentável, "compreendendo" as internalidades e externalidades positivas e negativas presentes no contexto da determinação da vazão ambiental.

\section{AGRADECIMENTOS}

O primeiro autor agradece ao CNPq/CT-HIDRO, pelo fomento à pesquisa no âmbito do doutorado, bem como ao Grupo do Projeto de Pesquisa "Integração dos Instrumentos de Outorga, Enquadramento e Cobrança para a Gestão das Águas Subterrâneas” - ASUB, financiado pelo MCT/FINEP/CT-HIDRO, pelo apoio técnico, banco de dados, ambiente físico de trabalho.

\section{REFERÊNCIAS}

ALBUQUERQUE, J. P. T.; RÊGO, J. C. Conceitos e definições para avaliação e gerenciamento conjunto de recursos hídricos superficiais e subterrâneos. In: SIMPÓSIO DE RECURSOS HÍDRICOS DO NORDESTE, 4., 1998, Campina Grande. Proceedings... Campina Grande: ABRH, 1998. 1 CD-ROM.

ALLEY, W. M.; RICHARD W. H.; LABAUGH, J. W.; REILLY, T. E. Flow and storage in groundwater systems: the dynamic nature of groundwater. Science, v. 296, p. 19851990, junho 2002.

http://dx.doi.org/10.1126/science.1067123

ALVES, M. H.; BERNARDO, J. M. Contribuição para uma metodologia de determinação do caudal ecológico em cursos e água temporários. In: CONGRESSO DA ÁGUA, 5., 2000, Lisboa Disponível em: <http://www.inag.pt/inag2004/port/a_intervencao/d_hidrico/pdf/estudos_amb/caudal_e co_cursos_temp.pdf $>$. Acesso: março 2011.

ASKOY, H. Markov chain-based modeling techniques for stochastic generation of daily intermittent streamflows. Advances in Water Resources, v. 26, p. 663-671, 2003. http://dx.doi.org/10.1016/S0309-1708(03)00031-9

BENDA, L.; POFF, L.; MILLER, D.; DUNNE, T.; REEVES, G.; PESS, G.; POLLOCK, M. The network dynamics hypothesis: how channel networks structure riverine hbitats. Bioscience, v. 54, n. 5, p 413-427, 2004. http://dx.doi.org/10.1641/0006-3568(2004)054[0413:TNDHHC]2.0.CO;2 
MEDEIROS, P. C.; SOUZA, F. A. S.; RIBEIRO, M. M. R. Aspectos conceituais sobre o regime hidrológico para a definição do hidrograma ambiental. Ambi-Agua, Taubaté, v. 6, n. 1, p. 131-147, 2011. (doi:10.4136/ambi-agua.179)

BENETTI, A. D.; LANNA, A. E. L; COBALCHINI, M. S. Metodologias para determinação de vazões ecológicas em rios. Revista Brasileira de Recursos Hídricos, v. 8, n. 2, p. 149-160, 2003.

BOULTON, J. A.; BOYERO L.; COVICH, A. P.; DOBSON, M.; LAKE S.; PEARSON, R. B. Are tropical streams ecologically different from temperate streams? Tropical Stream Ecology, p. 257-284, 2008. Disponível em: <http://eprints.jcu.edu.au/7493/>. Acesso: março 2011.

BRASIL. Lei Federal $\mathbf{n}^{\circ}$. 9.433, de 08 de janeiro de 1997. Instituiu a política nacional de recursos hídricos e cria o sistema nacional de gerenciamento de recursos hídricos. Disponível em: <http://www.cnrh-srh.gov.br/>. Acesso: 20 dez. 2005.

BRODIE, R.; SUNDARAM, B.; TOTTENHAM R.; HOSTETLER, S.; RANSLEY, T. An adaptive management framework for connected groundwater-surface water resources in Austrália. Bureau of Rural Sciences. 2007b. Disponível em: $<$ http://adl.brs.gov.au/brsShop/data/adaptivemgtframeworkgroundwatersurfacewater.pd f>. Acesso: março 2011.

BRODIE, R.; SUNDARAM, B.; TOTTENHAM R.; HOSTETLER, S.; RANSLEY, T. An overview of tools for assessing groundwater-surface water connectivity. Bureau of Rural Sciences. 2007a. Disponível em: < http://adl.brs.gov.au/brsShop/data/assessinggroundwatersurfacewaterconnectivity.pdf>. Acesso: março 2011.

BROWN, R. A.; PASTERNACK, G. B. Engineered channel controls limiting spawning habitat rehabilitation success on regulated gravel-bed rivers. Geomorphology, v. 97, p. 631-654, 2008.

http://dx.doi.org/10.1016/j.geomorph.2007.09.012

BRUNO, M. C.; LOFTUS W. F.; REID, J. W.; PERRY S. A. Diapause in copepods (Crustacea) from ephemeral habitats with different hydroperiods in Everglades National Park (Florida, U.S.A.). Hydrobiologia, v. 453/454, p. 295-308, 2001. http://dx.doi.org/10.1023/A:1013161210836

BUNN, S. E.; ARTHINGTON, A. H. Basic principles and ecological consequences of altered flow regimes for aquatic biodiversity. Environmental Management, v. 30, n. 4, p. 492-507, 2002.

http://dx.doi.org/10.1007/s00267-002-2737-0

BUSS, S.; ZUANSI, C.; CARDENAS, B.; FLECKENSTEIN, J.; HANNAH, D.; HEPPELL, K.; HULME, P. et al. The hyporheic handbook: a handbook on the groundwatersurface water interface and hyporheic zone for environment managers. Head of Science. 280p. 2009. Disponível em <www.hyporheic.net/Website\%20format\%20ch9.pdf>. Acesso: março 2011.

BURCHER, C. L. Using simplified watershed hydrology to define spatially explicit 'zones of influence'. Hydrobiologia, v. 618, p. 149-160, 2009.

http://dx.doi.org/10.1007/s10750-008-9572-0

CALLANAN, M.; BAARS, J. R.; KELLY-QUINN, M. Critical influence of seasonal sampling on the ecological quality assessment of small headwater streams. Hydrobiologia, v. 610, n. 1, p. 245-255, 2008.

http://dx.doi.org/10.1007/s10750-008-9439-4 
MEDEIROS, P. C.; SOUZA, F. A. S.; RIBEIRO, M. M. R. Aspectos conceituais sobre o regime hidrológico para a definição do hidrograma ambiental. Ambi-Agua, Taubaté, v. 6, n. 1, p. 131-147, 2011. (doi:10.4136/ambi-agua.179)

CARLISLE, D. M.; FALCONE, J.; WOLOCK, D. M.; MEADOR, M. R.; NORRIS, R. H. Predicting the natural flow regime: models for assessing hydrological alteration in streams. River Research and Applications. v. 26, p. 118 - 136, 2009.

CEY, E. E.; RUDOLPH, D. L.; PARKIN, G. W.; ARAVENA, R. Quantifying groundwater discharge to a small perennial stream in southern Ontario, Canada. Journal of Hydrology. v. 210, p. 21-37, 2008. http://dx.doi.org/10.1016/S0022-1694(98)00172-3

CHANG, F.; TSAI, M.; TSAI, W.; HERRICKS E. E. Assessing the ecological hydrology of natural flow conditions in Taiwan. Journal of Hydrology. v. 354, p. 75- 89, 2008. http://dx.doi.org/10.1016/j.jhydrol.2008.02.022

CHEN. X.; CHEN, D. Y.; CHEN, X. Simulation of baseflow accounting for the effect of bank storage and its implication in baseflow separation. Journal of Hydrology. v. 327, p. 539-549, 2006.

http://dx.doi.org/10.1016/j.jhydrol.2005.11.057

CIESIELKA, I. K.; BAILEY, R. C. Hierarchical structure of stream ecosystems: consequences for bioassessment. Hydrobiologia, v. 586, n. 1, p. 57-67, 2007.

http://dx.doi.org/10.1007/s10750-006-0481-9

CONSELHO NACIONAL DE DESENVOLVIMENTO CIENTÍFICO E TECNOLÓGICO CNPQ. Edital MCT/CNPq/CT-Hidro - $\mathbf{N}^{\mathbf{0}}$ 045/2006. 2006. Disponível em: < www.cnpq.br/editais/ct/2006/045.htm>. Acesso: março 2011.

COLLISCHONN, W.; SOUZA, C. F.; FREITAS, G. K.; PRIANTE, G. R.; AGRA, S. G.; TASSI, R. Em Busca do hidrograma ecológico. In: SEMINÁRIO BRASILEIRO DE RECURSOS HÍDRICOS, 16., 2005, João Pessoa. Proceedings... João Pessoa: ABRH, 2005. 1 CD-ROM.

COSTA, W. D.; ALBUQUERQUE, J. do P. T.; BRANCO, R. L. C.; MARANHÃO, C. M. L.; GOLDFABER, M. Estudo de caracterização e verificação da disponibilidade hídrica da vertente litorânea do estado da Paraíba: estudos hidrogeológicos. Relatório Final. Brasília: Ministério da Integração Nacional, 2007. Tomo I - Texto.

CRUZ, J. C. Disponibilidade hídrica para outorga: avaliação de aspectos técnicos e conceituais. 2001. Tese (Doutorado em Engenharia de Recursos Hídricos) Universidade Federal do Rio Grande do Sul, Porto Alegre, 2001.

CRUZ. R. C. Prescrição de vazão ecológica: aspectos conceituais e técnicos para bacias com carência de dados. 2005. Tese (Doutorado em Ecologia)_ - Instituto de Biociências, Universidade Federal do Rio Grande do Sul, Porto Alegre, 2005.

DAHL, M.; NILSSON B.; LANGHOFF J. H.; REFSGAARD J. C. Review of classification systems and new multi-scale typology of groundwater-surface water interaction. Journal of Hydrology, v. 344, p. 1-16, September 2007. http://dx.doi.org/10.1016/j.jhydrol.2007.06.027

DEITCH, M. J.; KONDOLF, G. M.; MERENLENDER, A. M. Hydrologic impacts of smallscale instream diversions for frost and heat protection in the California wine country. River Research and Applications, v. 25. p. 118-134, 2009a. 
MEDEIROS, P. C.; SOUZA, F. A. S.; RIBEIRO, M. M. R. Aspectos conceituais sobre o regime hidrológico para a definição do hidrograma ambiental. Ambi-Agua, Taubaté, v. 6, n. 1, p. 131-147, 2011. (doi:10.4136/ambi-agua.179)

DEITCH, M. J.; KONDOLF, G. M.; MERENLENDER, A. M. Surface water balance to evaluate the hydrological impacts of small instream diversions and application to the Russian River basin, California, USA. Aquatic conservation: marine and freshwater ecosystems, v. 19. p. 274-284, 2009b.

http://dx.doi.org/10.1002/rra.1100

EAMUS, D.; HATTON, T.; COOK, P.; COLVIN, C. Ecohydrology: vegetation function, water and resource management. Collingwood: CSIRO, 2006.

ECKSTEIN, Y.; ECKSTEIN, G. Transboundary aquifers: conceptual models for development of international law. Ground Water, v. 43, n. 5, p. 679-690, Sep./Oct., 2005. http://dx.doi.org/10.1111/j.1745-6584.2005.00098.x

FARIAS JÚNIOR, J. E. F.; CARVALHO, G. S.; AZEVEDO, J. P. S.; MAGALHAES, P. C. Determinação da demanda ecológica em pequenas bacias: caso da Bacia Hidrográfica do Rio Coruripe. In: SIMPÓSIO BRASILEIRO DE RECURSOS HÍDRICOS, 16. 2005, João Pessoa. Proceedings... João Pessoa: SBRH, 2005. 1 CD-ROM.

FARIAS JÚNIOR, J. E. F. Análise de metodologias utilizadas para a determinação da vazão ecológica. Estudo de caso: Rio Voruripe/AL e Rio Solimões/AM. 2006. Dissertação (Mestrado em Engenharia Civil) - Universidade Federal do Rio de Janeiro, , Rio de Janeiro, 2006.

FEITOSA, F.A.C. Pesquisa de água subterrânea. In: FEITOSA F. A. C.; MANOEL FILHO, J. Hidrogeologia: - conceitos e aplicações. Fortaleza: CPRM, LABHID-UFPE, 1997. 389p.

FERRAZ, F. F. B.; MORTATTI, J. Avaliação da relação entre distribuição espacial da precipitação e os componentes do escoamento em bacia tropical de Meso-escala, GeoUsp, São Paulo, 2003. p. 13. Disponivel em: <www.geografia.fflch.usp.br/publicacoes/Geousp/Geousp13/Geousp13G_Ferraz_Morta tti.htm>. Acesso: março 2011.

FITTS, C. Groundwater science. San Diego: Academic Press; Elsevier Science, 2002. 450p.

GALASSI, D. M. P. Groundwater copepods: diversity patterns over ecological and evolutionary scales. Hydrobiologia, v. 453/454, p. 227-253, 2001. http://dx.doi.org/10.1023/A:1013100924948

GAO, Y.; VOGEL, R. M.; KROLL, C. N.; POFF, N. L.; OLDEN, J. D. Development of representative indicators of hydrologic alteration. Journal of Hydrology, v. 374, p. 136-147, 2009.

http://dx.doi.org/10.1016/j.jhydrol.2009.06.009

GORE, J. A. Discharge measurements and streamflow analysis. In: HAUER, R.; LAMBERTI, G. A. Methods in stream ecology. 2. ed.. [S.l.]: Elsevier, 2007. p.51-77. http://dx.doi.org/10.1016/B978-012332908-0.50005-X

HAN, D.; HAMMOND, M. Recession curve estimation for storm event separations. Journal of Hydrology. v. 330, p. 573- 585, 2006. http://dx.doi.org/10.1016/j.jhydrol.2006.04.027

HARDISTY, J. Estuaries: monitoring and modeling the physical system. Malden: Blackwell Publishing, 2007. 157p.

http://dx.doi.org/10.1002/9780470750889 
MEDEIROS, P. C.; SOUZA, F. A. S.; RIBEIRO, M. M. R. Aspectos conceituais sobre o regime hidrológico para a definição do hidrograma ambiental. Ambi-Agua, Taubaté, v. 6, n. 1, p. 131-147, 2011. (doi:10.4136/ambi-agua.179)

HARVEY F. E.; SWINEHART J. B.; KURTZ, T. M. Sustenance of Nebraska's unique Sand Hills peatland fen ecosystems. Ground Water, v. 45, n. 2, p. 218-234, 2007. http://dx.doi.org/10.1111/j.1745-6584.2006.00278.x

HAYS, R. L.; ULLMAN, W. Direct determination of total and fresh groundwater discharge and nutrient loads from a sandy beachface at low tide (Cape Henlopen, Delaware). Limnology and oceanography. v. 52, n. 1, p. 240-247, 2007. http://dx.doi.org/10.4319/lo.2007.52.1.0240

HU, W.; WANG, G.; DENG, D.; LI, S. The influence of dams on ecohydrological conditions in the Huaihe River basin, China. Ecological Engineering, v. 33, p. 233-241, 2008. http://dx.doi.org/10.1016/j.ecoleng.2008.04.003

IDEIÃO, S. M. A.; COSTA, I. Y. L. G.; SANTOS, C. A. G. Análise da viabilidade do uso de água de chuva em condomínios horizontais através da Transformada Wavelet. In: SEMINÁRIO IBEROAMERICANO SOBRE SISTEMAS DE ABASTECIMENTO URBANO DE ÁGUA, 6., 2006, João Pessoa. Proceedings... João Pessoa: UFPB, 2006. v.1, p. 1-8. 1 CD-ROM.

JACOBSON, R. B.; GALAT, D. L. Flow and form in rehabilitation of large-river ecosystems: An example from the Lower Missouri River. Geomorphology, v. 77. p. 249-269, 2006. http://dx.doi.org/10.1016/j.geomorph.2006.01.014

JAIN, A.; SRINIVASULU, S. Integrated approach to model decomposed flow hydrograph using artificial neural network and conceptual techniques. Journal of Hydrology. v. 317, p. 291-306, 2006.

http://dx.doi.org/10.1016/j.jhydrol.2005.05.022

JØRGENSEN, S. E.; FATH, B.; BASTIANONI, S.; MARQUES, J.; MULLER, F.; NIELSEN, S. N. et al. Ecosystem principles have broad explanatory power in ecology. In: A new ecology:. systems perspective. Oxford: Elsevier, 2007. 289p.

KALBUS, E.; REINSTORF, F.; SCHIRMER, M. Measuring methods for groundwater, surface water and their interactions: a review. Hydrology and Earth System Sciences Discussions, v. 3, p. 1809-1850, 2006. http://dx.doi.org/10.5194/hessd-3-1809-2006

KING, A. J.; TONKIN, Z.; MAHONEY, J. Environmental flow enhances native fish spawning and recruitment in the Murray River, Australia. River Research and Applications, v. 25, p. 1205-1218, 2008. http://dx.doi.org/10.1002/rra.1209

LEAP, D. I. Geological occurrence of groundwater. In: DELLEUR, J. (Ed.). The handbook of groundwater engineering. Boca Raton: CRC Press, 1999.

LUZ, L. D.; AMORIN, R. B.; AMORIM, F. B.; MEDEIROS Y. Aplicabilidade do conceito de vazões ecológicas ao semi-árido brasileiro. In: CONFERÊNCIA INTERNACIONAL SOBRE ÁGUA EM REGIÕES ÁRIDAS E SEMIÁRIDAS, 2., 2007, Gravatá. Gravatá: ABRH, 2007.

LUZ, L. D.; SANTOS, P. F.; LORDELO, F.; GÓES, T. Fluxos de base: equipamento destinado a medições - desenvolvimento e primeiros experimentos. In: SIMPÓSIO DE RECURSOS HÍDRICOS DO NORDESTE, 10., novembro 2008, Salvador. Proceedings... Salvador: ABRH, 2008. 1 CD-ROM. 
MEDEIROS, P. C.; SOUZA, F. A. S.; RIBEIRO, M. M. R. Aspectos conceituais sobre o regime hidrológico para a definição do hidrograma ambiental. Ambi-Agua, Taubaté, v. 6, n. 1, p. 131-147, 2011. (doi:10.4136/ambi-agua.179)

MCCLAIN, M. E.; BOYER, E. W.; DENT, C. L.; GERGEL, S. E.; GRIMM, N. B.; GROFFMAN, P. M. Biogeochemical hot spots and hot moments at the interface of terrestrial and aquatic ecosystems. Ecosystems, v. 6, p. 301-312, 2003.

http://dx.doi.org/10.1007/s10021-003-0161-9

MALTCHIK, L. G. Ecologia de rios intermitentes tropicais. In: POMPEO, M. L. M. (Org.). Perspectivas da limnologia no Brasil. São Luís: União, 1999. p. 77-89.

MASIH, I.; AHMAD, M.; UHLENBROOK, S.; TURRAL, H.; KARIMI, P. Analysing streamflow variability and water allocation for sustainable management of water resources in the semi-arid Karkheh river basin, Iran. Physics and Chemistry of the Earth, v. 34, p. 329-340, 2009.

MEDEIROS, Y. D. P.; LUZ, L. D.; AMORIM, F. B.; GONÇALVES, M. S.; BERETTA, M. CAMPOS, V. P. et al.. Alocação de águas em bacias hidrográficas - uma abordagem ambiental. In: SIMPÓSIO DE RECURSOS HÍDRICOS DO NORDESTE, 8., 2006, Gravatá. Proceedings... Gravatá: ABRH, 2006. 1CD-ROM.

MEYER, A.; MEYER, E. I; MEYER, C. Lotic communities of two small temporary karstic stream systems (East Westphalia, Germany) along a longitudinal gradient of hydrological intermittency. Limnologica - Ecology and Management of Inland Waters, v. 33, p. 271-279, 2003.

MILLER, D. C.; ULLMAN. W. J. Ecological consequences of groundwater discharge to Delaware Bay, United States. Ground Water, v. 42, p. 959-970, 2004. http://dx.doi.org/10.1111/j.1745-6584.2004.tb02635.x

MINSHALL, G. W.; RUGENSKI, A. Riparian processes and interactions. In: HAUER, R.; LAMBERTI, G. A. Methods in stream ecology. 2. ed.. [S.l.]: Elsevier, 2007. p. 721742.

MOLIERE, D. R.; LOWRY, J. B. C.; HUMPHREY, C. L. Classifying the flow regime of data-limited streams in the wet-dry tropical region of Australia. Journal of Hydrology, v. 367, p. 1-13, 2009.

http://dx.doi.org/10.1016/j.jhydrol.2008.12.015

MONTGOMERY, D. R. Process domains and the river continuum. Journal of the American Water Resources Association, v. 35, n. 2, p. 397-410, 1999.

http://dx.doi.org/10.1111/j.1752-1688.1999.tb03598.x

NAIMAN, R. J.; LATTERELL, J. J.; PETTIT, N. E.; OLDEN, J. D. Flow variability and the biophysical vitality of river systems. Comptes Rendus Geoscience, v. 340, n. 9/10, p. 629-643, 2008.

http://dx.doi.org/10.1016/j.crte.2008.01.002

NATHAN, R. J.; MCMAHON, T. A. Evaluation of automated techniques for baseflow and recession analysis. Water Resources Research, v. 26, n. 7, p. 1465-1473, 1990. http://dx.doi.org/10.1029/WR026i007p01465

NEUBAUER, C. P.; HALL, G. B.; LOWE, E. F.; ROBISON, C. P.; HUPALO, R. B.; KEENAN, L. W. Minimum flows and levels method of the St. Johns River Water Management District, Florida, USA. Environmental Management, v. 42, p. 11011114, 2008. http://dx.doi.org/10.1007/s00267-008-9199-y 
MEDEIROS, P. C.; SOUZA, F. A. S.; RIBEIRO, M. M. R. Aspectos conceituais sobre o regime hidrológico para a definição do hidrograma ambiental. Ambi-Agua, Taubaté, v. 6, n. 1, p. 131-147, 2011. (doi:10.4136/ambi-agua.179)

PETTS, G. E. A perspective on the abiotic processes sustaining the ecological integrity of running waters. Hidrobyologia, v. 422-423, p.15-27, 2000.

http://dx.doi.org/10.1023/A:1017062032685

PINDER, G. F.; CELIA, M. A. Subsurface hydrology. Hoboken: John Wiley \& Sons, 2006. 468p.

http://dx.doi.org/10.1002/0470044209

POFF, L. N.; ALLAN, J. D.; BAIN, M.B.; KARR, J. R.; PRESTEGAARD, K. L.; RICHTER, $B$. D. et al. The natural flow regime: a paradigm for river conservation and restoration. BioScience, v. 47, n. 11, p. 769-784, 1997.

http://dx.doi.org/10.2307/1313099

POSTEL, S.; RICHTER, B. Rivers for life: managing water for peaple and nature. Washington, D.C.: Island Press, 2003. 253 p.

QUEIROZ, M. M. F. de; BOAS, M. A. V.; OLIVEIRA, L. M. Análise de vazões mínimas Q1,10 e Q7,10 de rios do Paraná através da distribuição Gev. In: SIMPÓSIO BRASILEIRO DE RECURSOS HÍDRICOS, 16., 2005, João Pessoa. Proceedings... João Pessoa: ABRH, 2005. 1CD-ROM.

REID, J. W. A human challenge: discovering and understanding continental copepod habitats. Hydrobiologia, v. 453/454, p. 201-226, 2001. http://dx.doi.org/10.1023/A:1013148808110

RENSCHLER, C. S.; DOYLE, M. W.; THOMS, M. Geomorphology and ecosystems: challenges and keys for success in bridging disciplines. Geomorphology, v. 89. p. 1-8, 2007. http://dx.doi.org/10.1016/j.geomorph.2006.07.011

ROSENBERRY, D. O. A seepage meter designed for use in flowing water. Journal of Hydrology. v. 359, p. 118- 130, 2008.

http://dx.doi.org/10.1016/j.jhydrol.2008.06.029

SANDERSON, J. S.; KOTLIAR, N. B.; STEINGRAEBER, D. A.; BROWNE, C. Simulated natural hydrologic regime of an intermountain playa conservation site. Wetlands, v. 28, n. 2, p. 363-377, 2008.

http://dx.doi.org/10.1672/07-76.1

SANTOS, V. S.; VIEIRA, A. S.; CURI, W. F.; CURI, R. C. Análise de metodologias de outorga dos direitos de uso da água para a sub-bacia do Rio Piancó - PB. In: SIMPÓSIO DE RECURSOS HÍDRICOS DO NORDESTE, 8., 2006, Gravatá. Proceedings... Gravatá: ABRH, 2006. 1 CD-ROM.

SHAW, E. M. Hydrology in practice. 3. ed. British Library, 2005. 613p

SILVA, F. F.; LORDELLO NETO, A. V.; CARDOSO, E. R. Vazão mínima, ecológica ou remanescente no Estado da Bahia. In: SIMPÓSIO DE RECURSOS HÍDRICOS DO NORDESTE, 8., 2006, Gravatá. Proceedings... Gravatá: ABRH, 2006. 1CD-ROM.

SILVA, F. C. Análise integrada de usos de água superficial e subterrânea em macroescala numa bacia hidrográfica: o caso do Alto Rio Paranaíba. 2007. 188f. Dissertação (Mestrado em Recursos Hídricos) - Instituto de Pesquisas Hidráulicas, Universidade Federal do Rio Grande do Sul, Porto Alegre, 2007. 
MEDEIROS, P. C.; SOUZA, F. A. S.; RIBEIRO, M. M. R. Aspectos conceituais sobre o regime hidrológico para a definição do hidrograma ambiental. Ambi-Agua, Taubaté, v. 6, n. 1, p. 131-147, 2011. (doi:10.4136/ambi-agua.179)

SMAKHTIN, V. U. Low flow hydrology: a review. Journal of Hydrology, v. 240, p.147186, 2001. http://dx.doi.org/10.1016/S0022-1694(00)00340-1

TALLAKSEN, L. M. A review of baseflow recession analysis. Journal of Hydrology. v. 165, p. 349-370, 1995. http://dx.doi.org/10.1016/0022-1694(94)02540-R

TOOD, D. K. Groundwater hydrology. 2. ed. [S.l.]: John Wiley \& Sons, 1987. 222p.

TUCCI, C. E. M. Escoamento superficial. In: TUCCI, C. E. M. (Org.). Hidrologia ciência e aplicação. Porto Alegre: Ed. Da Universidade; ABRH; EDUSP, 2003.

TUCCI, C. E. M. Hidrograma ambiental. Outubro 2009. Disponível em: <http://blog.rhama.net/2009/10/04/hidrograma-ambiental/>. Acesso: março 2011.

VANNOTE, R. L.; MINSHALL, G. W.; CUMMINS, K. W.; SEDELL, J. R.; CUSHING, C. E. The river continuum concept. Canadian Journal of Fisheries and Aquatic Sciences, v. 37, p. 130-137, 1980. http://dx.doi.org/10.1139/f80-017

WEBER K. A.; PERRY, R. G. Groundwater abstraction impacts on spring flow and base flow in the Hillsborough River Basin, Florida, USA. Hydrogeology Journal, v. 14, p. 1252-1264, 2006. http://dx.doi.org/10.1007/s10040-006-0040-5

WELLER, D. E.; JORDAN, T. E.; CORRELL, D. L. Heuristic models for material discharge from landscapes with riparian buffers. Ecological Applications: v. 8, n. 4, p. 11561169, 1998. http://dx.doi.org/10.1890/1051-0761(1998)008[1156:HMFMDF]2.0.CO;2

WEN, L. Reconstruction natural flow in a regulated system, the murrumbidgee river, australia, using time series analysis. Journal of Hydrology. v. 364. p. 216-226, 2009. http://dx.doi.org/10.1016/j.jhydrol.2008.10.023

WINTER, T. C.; HARVEY, J. W.; FRANKE, O. L.; ALLEY, W. M. Ground water and surface water a single resource. U. S. Geological Survey Circular, n. 1139, 1998.

ZEDLER, J. B. Handbook for restoring tidal wetlands. Boca Raton: CRC Press; Marine Science Series, 2001.

ZEILHOFERA, P.; MOURA, R. M. Hydrological changes in the northern Pantanal caused by the Manso dam: impact analysis and suggestions for mitigation. Ecological Engineering. v. 35, p. 105-117, 2009.

http://dx.doi.org/10.1016/j.ecoleng.2008.09.011 